This is the accepted version of the following article:

Calabretta M.M., Álvarez-Diduk R., Michelini E., Roda A., Merkoçi A.. Nano-lantern on paper for smartphone-based ATP detection. Biosensors and Bioelectronics, (2020). 150. 111902: - . 10.1016/j.bios.2019.111902,

which has been published in final form at https://dx.doi.org/10.1016/j.bios.2019.111902 @ https://dx.doi.org/10.1016/j.bios.2019.111902. This manuscript version is made available under the CC-BY-NC-ND 4.0 license http://creativecommons.org/licenses/by-nc-nd/4.0/ 


\section{Nano-lantern on paper for smartphone-based ATP detection}

Maria Maddalena Calabretta ${ }^{\mathrm{a}, \mathrm{b}}$, Ruslan Álvarez-Diduk ${ }^{\mathrm{b}}$, Elisa Michelini ${ }^{\mathrm{a}, \mathrm{c}, \mathrm{d} *}$, Aldo Roda ${ }^{\mathrm{a}, \mathrm{c} *}$, Arben $\operatorname{Merkoçi~}^{\mathrm{b}, \mathrm{e} *}$

aDepartment of Chemistry “G. Ciamician”, University of Bologna, Via Selmi 2, 40126 Bologna, Italy;

${ }^{\mathrm{b}}$ Nanobioelectronics and Biosensors Group, Catalan Institute of Nanoscience and Nanotechnology

(ICN2), CSIC. The Barcelona Institute of Science and Technology, Campus UAB, Bellaterra, 08193,

Barcelona, Spain.

${ }^{\mathrm{c}}$ INBB, Istituto Nazionale di Biostrutture e Biosistemi, 00136 Rome, Italy;

${ }^{\mathrm{d} H e a l t h}$ Sciences and Technologies-Interdepartmental Center for Industrial Research (HST-ICIR), University of Bologna, via Tolara di Sopra 41/E 40064, Ozzano dell'Emilia, Bologna, Italy;

${ }^{\text {e}}$ Catalan Institution for Research and Advanced Studies (ICREA), Pg. Lluís Companys 23, 08010 Barcelona, Spain.

*Corresponding authors:

Prof. Arben Merkoçi

Nanobioelectronics \& Biosensors Group

Institut Català de Nanotecnologia (ICN)

Campus de la UAB - CIN2

08193 Bellaterra (Barcelona) Spain

Tel: +34937374604

E-mail: arben.merkoci@icn.cat

Prof. Aldo Roda

Dept. of Chemistry "G. Ciamician"

University of Bologna, Via Selmi 2

40126-Bologna, Italy

Tel/Fax +39 0516364166

Email: aldo.roda@unibo.it

Prof. Elisa Michelini

Dept. of Chemistry "G. Ciamician"

University of Bologna, Via Selmi 2

40126-Bologna, Italy

Tel/Fax +39051343398

Email: elisa.michelini8@unibo.it 


\begin{abstract}
ATP-driven bioluminescence relying on the D-luciferin-luciferase reaction is widely employed for several biosensing applications where bacterial ATP detection allows to verify microbial contamination for hygiene monitoring in hospitals, food processing and in general for cell viability studies. Several ATP kit assays are already commercially available but an user-friendly ATP biosensor characterized by low-cost, portability, and adequate sensitivity would be highly valuable for rapid and facile on site screening.

Thanks to an innovative freeze-drying procedure, we developed a user-friendly, ready-to-use and stable ATP sensing paper biosensor that can be combined with smartphone detection. The ATP sensing paper includes a lyophilized "nano-lantern" with reaction components being rapidly reconstituted by $10 \mu \mathrm{L}$ sample addition, enabling detection of $10^{-14}$ moles of ATP within 10 minutes. We analysed urinary microbial ATP as a biomarker of urinary tract infection (UTI), confirming the capability of the ATP sensing paper to detect the threshold for positivity corresponding to $10^{5}$ colonyforming units of bacteria per $\mathrm{mL}$ of urine.
\end{abstract}

Keywords: bioluminescence, ATP biosensor, luciferase, smartphone, paper-based biosensor 


\section{Introduction}

The detection of adenosine-5'-triphosphate (ATP), the energy-carrying molecule found in the cells of all living organisms, represents a well-established approach to detect living cells, which is of paramount importance in several fields including environmental monitoring, detection of food microbial contaminants, and hygiene monitoring (Roda et al., 2004; Huang et al. 2015; Nascetti et al. 2019; Dostálek and Brányik, 2005). Moreover, ATP levels are related to several diseases and pathological states, such as Alzheimer's disease, ischemia, hypoxia (Onyango et al., 2016; Zhang et al., 2015; Akbara et al., 2016). Thanks to its catalytic activity, luciferase from North American firefly Photinus pyralis (Luc) has been widely employed to detect ATP with excellent sensitivity (Branchini and Southworth, 2017; Morciano et al., 2017). It produces light by oxidizing D-luciferin $\left(\mathrm{LH}_{2}\right)$ in the presence of ATP, $\mathrm{Mg}^{2+}$ and $\mathrm{O}_{2}$ according to the following reactions:

$$
\begin{aligned}
& L u c+\mathrm{LH}_{2}+A T P \stackrel{\mathrm{Mg}^{2+}}{\leftrightarrows} \mathrm{Luc} \cdot \mathrm{LH}_{2}-A M P+P p i \\
& \mathrm{Luc} \cdot \mathrm{LH}_{2}-\mathrm{AMP}+\mathrm{O}_{2} \rightarrow \mathrm{Luc} \cdot \text { Oxyluciferin } \\
& *
\end{aligned}
$$

In the first step, D-luciferin in the presence of ATP, is converted into luciferyl adenylate (eq. 1), the central intermediate in the BL reaction. In the presence of molecular oxygen, around $80 \%$ of adenylate is oxidized via a single electron-transfer mechanism (Branchini et al., 2015) into peroxide leading to production of oxyluciferin and emission of a photon (eq. 3).

The ATP concentration is therefore related to the bacterial counts assuming a fix and constant intracellular concentration thus allowing the extrapolation to the number of bacteria cells.

Several attempts were aimed at improving the assay performance by increasing luciferase stability, by modifying the emission wavelength for multiplexing, by improving the quantum yield of the light producing reaction by mutating the sequence encoding for the luciferase enzyme (Branchini et al., 2007), by adding activators or stabilizers (Lonshakova-Mukina et al., 2005), and by obtaining D- 
luciferin analogues. A remarkable limit of detection of 1 amol of ATP, corresponding to a single bacterial cell, can be achieved exploiting the luciferase/luciferin reaction in solution using benchtop instrumentation for light detection (Lundin, 1999; Adachi et al., 1993; Yamana et al., 2003; Satoh et al., 2004).

Thanks to the high sensitivity and intrinsic specificity of the reaction, several bioluminescence-based assays have been developed to monitor air and surface cleanliness, and for food and pharmaceutical quality controls (Squirrel et al., 2002; Aycicek et al., 2006; Dostalek and Branyik, 2005). Exploiting the measurement ATP as metabolic activity of microbial contamination, urine screen with bioluminescence detection has been widely reported in the literature (Thore et al., 1983; Ivančić et al., 2008; Feng et al., 2014; ). In urinary tract infection (UTI) disease, increased urinary ATP levels are associated to bacterial invasion and cells inflammation with high levels found in patients with interstitial cystitis and acute UTI (Lundin et al., 1989). Although an accurate diagnosis of UTI relies on the identification of bacteria, a urinary bacteria count of $\geq 10^{5}$ colony forming units/mL $(\mathrm{CFU} / \mathrm{mL})$ is commonly accepted as the threshold for bacteriuria positivity (http://www.uroweb.org/nc/professional-resources/guidelines/online/, Schmiemann et al., 2008).

The use of ATP-driven bioluminescence has been widely used in laboratory-based settings for the analysis of urine samples, however the implementation of this assay in a biosensor format for pointof-care and field analysis is limited by the relatively low stability of luciferase (Ribeiro et al., 1998). Indeed, enzyme solutions are easily inactivated and need storage conditions between +4 and $+8^{\circ} \mathrm{C}$. Conversely, by immobilizing reagents (both enzyme and substrate) on a solid support (such as paper) it is possible to improve the long-term stability of enzymes without special storage conditions (Kratasyuk and Esimbekova, 2003; Esimbekova et al., 2009). Therefore, several efforts have been focused on the immobilization of luciferase on different substrates including polysaccharide carriers (Ugarova et al, 1982), nylon tubes (Carrea et al., 1986), alkyl-substituted Sepharose 4B (YousefiNejad et al, 2007), and poly(vinyl alcohol-co-ethylene) (PVA-coPE) nanofiber membranes (Wang et al, 2015). Paper provides a valuable substrate for sensing applications because it represents an ideal 
versatile and eco-friendly platform to develop low-cost portable devices for diagnostic and field deployment monitoring (Wu et al., 2018; Arduini et al., 2019). Its attractive features such as hydrophilicity, biocompatibility, biodegradability, and porosity combined to the easiness of functionalization and production (López-Marzo and Merkoçi, 2016) enable facile coupling with portable detectors and smartphones (Álvarez-Diduk et al., 2017, Capoferri et al., 2018), thus ensuring wide applicability in several fields including clinical diagnosis, food quality control and environmental monitoring (López-Marzo and Merkoçi, 2016; Calabria et al., 2017). Paper has been seldom applied as supporting layer for ATP analysis (Zhang et al, 2014; Ge et al., 2013), however detection limits do not generally compete with laboratory-based assays and solution-based biosensors (Nascetti et al., 2019; Lundin, 1999; Adachi et al., 1993; Yamana et al., 2003; Satoh et al., 2004).

Besides, the low-light emitted by the bioluminescent reaction may represent a challenge for the light collection efficiency of portable detectors such as charge coupled devices (CCDs), complementary metal-oxide semiconductors (CMOSs) and smartphone-integrated CMOSs. Innovative advances in light detection technology have made it possible the implementation of several bioluminescent biosensors relying on luciferase into portable analytical devices (Cevenini et al., 2016; Michelini et al., 2019; Lopreside et al., 2019). Recently a miniaturized system has been reported combining a 3D printed microfluidic chip and silicon photomultiplier (Santangelo et al, 2018). This continuous-flow system provided almost real-time response and a remarkable limit of detection of $8 \mathrm{nM}$ for ATP, showing analogous dynamic range as a benchtop luminometer. The feasibility of immobilizing luciferase/D-luciferin onto filter paper was also demonstrated (Nguyen et al, 2018). However, due to the loss of activity after immobilization, large amounts of enzyme are generally used to achieve adequate analytical performance. This inevitably increases total assay cost and reduces economical feasibility of commercial exploitation.

To address such limitations and provide a user-friendly platform we developed a new lyophilisation strategy that enable to immobilize luciferase and reagents required for bioluminescent reaction directly on paper with higher reproducibility and enzyme stability than previously reported methods 
(Yousefi-Nejad et al, 2007; Wang et al, 2015). By exploiting paper as support a wax-printed nitrocellulose paper biosensor was developed using smartphone photocamera as light detector (Fig. 1).

To fully exploit the combination with smartphone, we selected high-performance back-sideilluminated (BSI) sensors characterized by higher sensitivity than previous models (Kim et al., 2017). We investigated the analytical performance and robustness of the newly developed lyophilization procedure with an intra- and inter-laboratory validation. We also investigated the stability of the luciferase/luciferin lyophilized on paper at different storage conditions. As proof-of-concept, urine samples spiked with Escherichia coli were analysed with the device, demonstrating the suitability of the device for detecting bacterial ATP for UTI diagnostics.

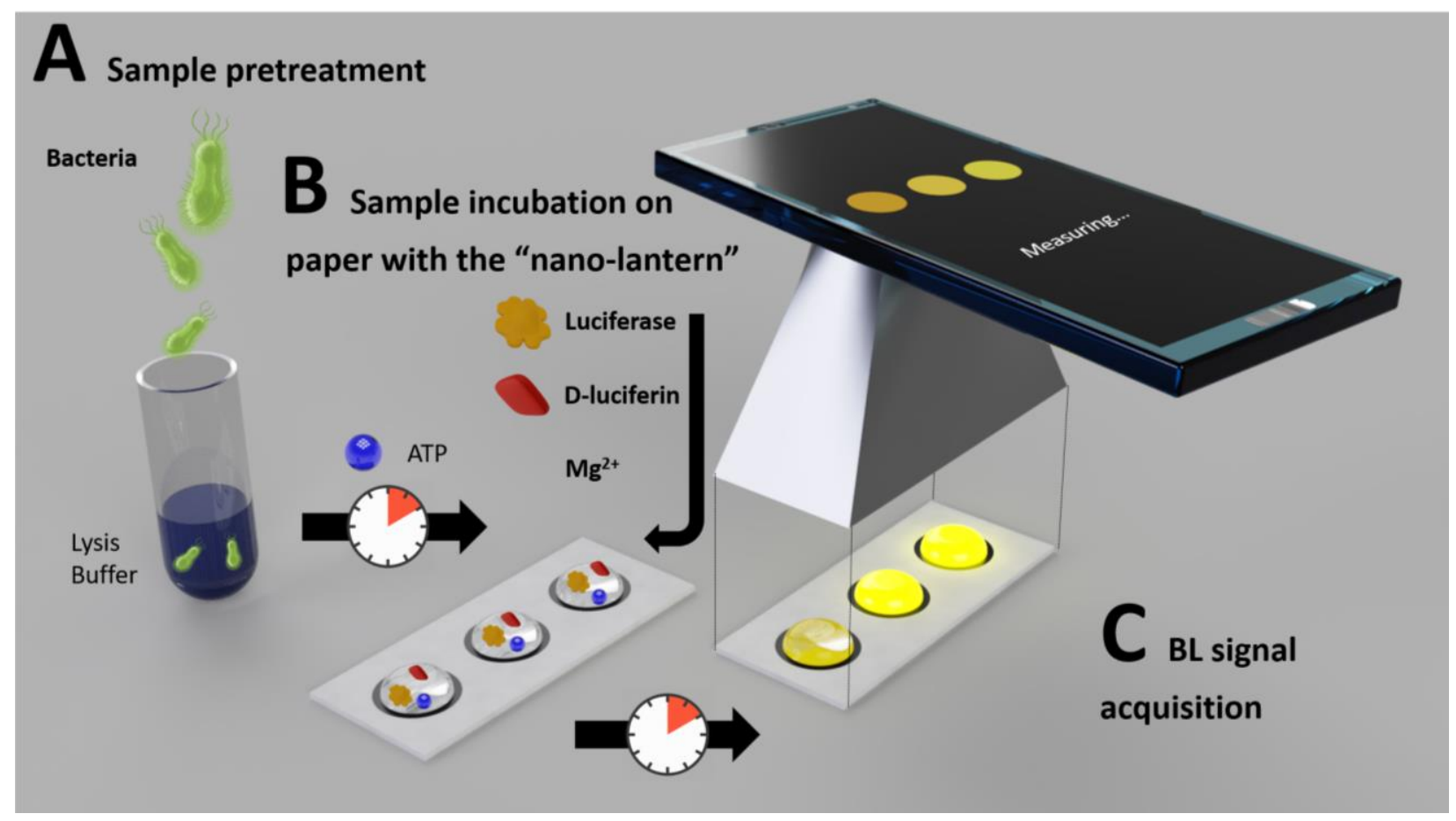

Fig. 1. Schematic representation of the optimized ATP sensing paper assay.

\section{Material and Methods}

\subsection{Reagents and instrumentation}

ATP Bioluminescence Assay Kit HS II including lyophilized luciferase reagent (LR) containing both 
luciferase and D-luciferin, lyophilized ATP, Dilution buffer (DB) and Lysis buffer was from Roche (Sigma-Aldrich). B-PER Bacterial Protein Extraction Reagent was from Thermo Fischer Scientific and bacterial strain JM109 was from Promega (Madison, WI, USA). Cryoprotectant R18 medium was prepared dissolving $7.5 \mathrm{~g}$ tryptone, $100 \mathrm{~g}$ sucrose, $50 \mathrm{~g}$ bovine serum albumin (BSA) in $1 \mathrm{~L}$ milliQ water. Wax printing technology was used for creating $5 \mathrm{~mm}$ wells on nitrocellulose paper from Millipore (HFC1800425) using a Color Qube 8570 wax printer (Xerox, Wethersfield, CT).

Freeze-dry analytical instruments Alpha 1-2 LDplus (Martin Christ Gefriertrocknungsanlagen $\mathrm{GmbH}$ ) and Cryodos-50 (Telstar Industrial, S.L.) were used to lyophilize LR.

Emission spectra and kinetic measurements were performed with Varioskan Flash multimode reader (ThermoFisher Scientific). Samsung Galaxy S7 integrated camera (12MP f/1.7, 1.4 $\mu$ pixel size, 1/2.6in sensor size, phase detect autofocus, OIS, dual-pixel sensor Samsung ISOCELL S5K2L1 or Sony Exmor RS IMX260), Oneplus5 smartphone (Oneplus, Shenzhen, China) equipped with an integrated camera (1/2.8" 16MP Sony IMX 398 sensor, 1.12 um pixel size and F1.7 aperture), and ATIK 383L cooled camera (ATIK Cameras, New Road, Norwich), equipped with a high resolution monochrome CCD sensor (Kodak KAF 8300, sensor size 17.6 x $13.52 \mathrm{~mm}$ ), were used as portable detectors.

\section{Preparation of ready-to-use ATP sensing paper}

Lyophilized luciferase/D-Luciferin reagent was prepared using DB according to the manufacturer instructions. ATP solutions, from $1.65 \times 10^{-3}$ to $1.65 \times 10^{-15} \mathrm{M}$, were prepared with serial dilutions from an ATP stock solution (16.5 x 10 $10^{-3} \mathrm{M}$ in DB).

An easy alternative protocol to freeze-drying LR on nitrocellulose paper was developed for obtaining a ready-to-use ATP sensing paper. Circular areas with a diameter of $5 \mathrm{~mm}$ were designed using Corel draw X6 (Corel Corporation, Ottawa, Canada) and created by wax printing technology using a nitrocellulose paper pad and the printer Xerox ColorQube 8570DN. An internal 3D-wax structure confining spot was obtained through a heating process $\left(2 \mathrm{~min}\right.$ at $\left.84^{\circ} \mathrm{C}\right)$. Then, the paper was sterilized 
using UV lamp for $5 \mathrm{hrs}$. For achieving the best lyophilization condition, different volumes of cryoprotector R18 medium $(1,2,3$, or $4 \mu \mathrm{L})$ were mixed to $12 \mu \mathrm{L}$ of $\mathrm{LR}$, placed on the discs and frozen directly in the vacuum drying chamber at $-50^{\circ} \mathrm{C}$. The vacuum drying chamber was gently opened in order to complete successive freeze-drying steps at $1.0 \mathrm{mBar}$ for $4 \mathrm{hrs}$. Same products were obtained with the standard freeze-drying procedure. Ten ATP sensing papers obtained with the new procedure were compared to ten ATP sensing papers obtained with the standard procedure after reconstitution of the $\mathrm{LR}$ with $8 \mu \mathrm{L} \mathrm{DB}$ and the addition of $2 \mu \mathrm{L}$ of ATP solution $1.65 \mathrm{mM}$. BL intensities were measured every 10min for 40 min with Samsung Galaxy S7 with $10 \mathrm{sec}$ integration and ISO 800 and analysed with Image J Software. Results were obtained with Graph Pad Prism using a second order polynomial (quadratic) curve. At the end of the procedure, ATP sensing papers were stored at either +4 or $-20^{\circ} \mathrm{C}$.

Characterization of lyophilized luciferase reagents on nitrocellulose paper disc: emission kinetics and emission spectra

Nitrocellulose discs were cut and lyophilized as described in "Preparation of ready-to-use ATP sensing paper". At the end of the procedure the discs were transferred to a black 96-well microplate and emission kinetic and spectra were measured with a Varioskan Flash multimode reader (Thermo Fisher Scientific) after the reconstitution of the lyophilized LR with $8 \mu \mathrm{L}$ of DB and the addition of $2 \mu \mathrm{L}$ of $1.65 \mathrm{mM}$ ATP, corresponding to $3.3 \times 10^{-9}$ moles. BL emission spectra were recorded in the range 450 - $750 \mathrm{~nm}$ (2-nm intervals, 200-ms integration time). Emission kinetics were acquired for $25 \mathrm{~min}$ with an integration time of $200 \mathrm{~ms}$.

Design and optimization the ATP sensing paper 
Different parameters and conditions were optimized including the solution used to rehydrate the LR reagents (DB or bi-distilled water), buffer volumes (from 6 to $12 \mu \mathrm{L}$ ) used for the reconstitution of $\mathrm{LR}$, the temperature $\left(20^{\circ} \mathrm{C}\right.$ to $\left.37^{\circ} \mathrm{C}\right)$, and incubation time with the sample (from 5 to $20 \mathrm{~min}$ ).

In the designed configuration, the ATP sensing paper includes 24 circular areas $(21$ microwells for ATP calibration curve and 3 microwells for the sample), each one representing a microwell in which the BL reaction occurs. For calibration curves, the assay procedure starts by depositing $8 \mu \mathrm{L}$ of $\mathrm{DB}$ for the reconstitution of LR. A $2 \mu \mathrm{L}$-volume of ATP solution in DB (concentration range from $1.64 \mathrm{x}$ $10^{-3}$ to $1.64 \times 10^{-9} \mathrm{M}$ ) is added to each microwell and incubated for $10 \mathrm{~min}$ at $22^{\circ} \mathrm{C}$. The ATP sensing paper is then transferred into a dark box, the luminescent signals are acquired with smartphone OnePlus 5 camera integrating BL signals for $30 \mathrm{sec}$ with ISO 3200 or with Samsung Galaxy S7 camera integrating BL signals for $10 \mathrm{sec}$ with ISO 800. Limit of detection was calculated as ATP concentration that corresponds to the blank plus three times the standard deviation. All measurements were performed in triplicate and repeated with different ATP sensing papers at least three times.

\section{Analytical performance of the ATP sensing paper with different light detectors}

Different light detectors including a standard benchtop luminometer, and portable CCD and smartphone integrated CMOSs were used to investigate the feasibility of using the ATP sensing paper in different settings. Varioskan Flash multimode reader was first used for evaluating the sensitivity of ATP sensing paper (concentration range from $1.64 \times 10^{-7}$ to $1.64 \times 10^{-15} \mathrm{M}$ ). BL signals were acquired for 30 min with an integration time of $200 \mathrm{~ms}$. BL images of ATP sensing paper (concentration range from $1.64 \times 10^{-3}$ to $1.64 \times 10^{-9} \mathrm{M}$ ) were also obtained with a cooled portable CCD camera (ATIK 383L+ mono cromo CCD) integrating BL signals from 5 to $60 \mathrm{sec}$ at $+5^{\circ} \mathrm{C}$. Two smartphones, i.e., Samsung Galaxy S7 and Oneplus 5, were selected to detect BL signals of ATP sensing paper: emission kinetics were measured for $40 \mathrm{~min}$ and images were taken for $10 \mathrm{sec}$ and 30 sec with different sensitivity settings, from ISO 100 to ISO 3200, after addition of serial dilutions of ATP (concentration range from $1.64 \times 10^{-3}$ to $1.64 \times 10^{-9} \mathrm{M}$ ). ImageJ software (National Institutes of 
Health, Bethesda, MD) and GraphPad Prism v.5 (GraphPad Software, La Jolla, USA) were used to analyse and to plot the data, respectively. Limit of Detection (LOD) was calculated as the blank plus three times the standard deviation. All measurements were performed in triplicate and repeated with different ATP sensing paper at least three times.

\section{Stability studies of ATP sensing paper}

Stability was investigated for $48 \mathrm{hrs}$ by measuring BL signals of ATP sensing paper obtained with standard and alternative procedures stored at room temperature, $+4^{\circ} \mathrm{C}$ and $-20^{\circ} \mathrm{C}$, and for 80 days with ATP sensing papers stored at $-20^{\circ} \mathrm{C}$ obtained with alternative procedure. $\mathrm{BL}$ intensities were acquired with a smartphone camera (Samsung Galaxy S7, $10 \mathrm{sec}$ ISO800) after reconstitution of the lyophilized reagents with $8 \mu \mathrm{L}$ of $\mathrm{DB}$ and the addition of $2 \mu \mathrm{L}$ of ATP $\left(1.65 \times 10^{-3} \mathrm{M}\right)$. All measurements were performed in triplicate.

\section{Inter-laboratory validation}

The study was performed with ATP sensing papers obtained with the optimized alternative freezedrying process using two different freeze-drying analytical instruments, Cryodos-50 located at the Institut Catala de Nanociencia i Nanotecnologia ICN2, Bellaterra, Barcellona, Spain and Alpha 1-2 LDplus located at the Department of Chemistry "Giacomo Ciamician”, University of Bologna, Italy. ATP sensing papers obtained as described in "Preparation of ready-to-use ATP sensing paper", were used for obtaining ATP calibration curves after the reconstitution of the lyophilized LR with $8 \mu \mathrm{L}$ of DB and the addition of $2 \mu \mathrm{L}$ ATP solutions (concentration range $1.64 \times 10^{-3}$ to $1.64 \times 10^{-9} \mathrm{M}$ ). $\mathrm{BL}$ measurements were performed with the same smartphone camera (Samsung Galaxy S7) integrating BL signals for $10 \mathrm{~s}$ with ISO 800. ImageJ software was used for analyzing BL images and all measurements were performed in triplicate. 
For analysis of ATP content in Escherichia coli, a glycerol stock of JM109 cells was used to inoculate in $5 \mathrm{~mL}$ cultures in Luria- Bertani Broth (LB). Cells were incubated overnight at $37^{\circ} \mathrm{C}$ with orbital shaking of $300 \mathrm{rpm}$ to optical density (OD) of 0.8 . A preliminary dilution in $5 \mathrm{~mL}$ of LB to reach $10^{8}$ $\mathrm{CFU} / \mathrm{mL}$ was performed followed by centrifugation for $10 \mathrm{~min}$ at 3500 . The supernatant was removed to eliminate extracellular ATP and the pellet was resuspended in $250 \mu \mathrm{L}$ of DB. Cell suspension was serially diluted in DB (concentration range of $5 \times 10^{4}-5 \times 10^{8} \mathrm{CFU} / 250 \mu \mathrm{L}$ ) and each solution was lysed with $250 \mu \mathrm{L}$ of BPER reagent. After 10 min incubation time $10 \mu \mathrm{L}$ of each lysed, corresponding to $10^{-15}-10^{-11}$ moles of ATP, was pipetted in triplicate on the ATP sensing paper and incubated for $10 \mathrm{~min}$ at room temperature. BL measurements were performed with OnePlus 5 acquiring BL signals for $30 \mathrm{sec}$ with ISO 3200. The images were analysed with ImageJ Software.

\section{Real samples analysis}

To explore the applicability of ATP sensing paper for analysis of ATP content in bacterial cells, $E$. coli was used as model bacterium. An overnight culture was grown in $5 \mathrm{~mL}$ of Luria Bertani broth at $37^{\circ} \mathrm{C}$ with orbital shaking of $300 \mathrm{rpm}$ to optical density (OD600) of 0.8 . For simulating the UTI, $E$. coli suspension was diluted in $10 \mathrm{~mL}$ of urine to obtain a concentration of $10^{5} \mathrm{CFU} / \mathrm{mL}$. Subsequently, cells were harvested by centrifugation $(3500 \mathrm{~g}$ for $10 \mathrm{~min}$ ) and the pellet was resuspended with 50 $\mu \mathrm{L}$ of DB. To select an efficient bacterial cell lysis method, several parameters were optimized such as the incubation time (from 5 to $30 \mathrm{~min}$ ), the incubation temperature $\left(22\right.$ and $37^{\circ} \mathrm{C}$ ) and with or without constant agitation (200-300 rpm). Cells lysis was carried out for 10 min adding $50 \mu \mathrm{L}$ of BPER buffer at $22{ }^{\circ} \mathrm{C}$. Finally, a volume of $10 \mu \mathrm{L}$ was pipetted onto the ATP sensing paper and incubated for $10 \mathrm{~min}$ at room temperature. BL intensities were acquired with OnePlus 5 camera (30sec integration time, ISO 3200) and BL signals were analysed with ImageJ software.

\section{Results and discussion}

ATP sensing paper design and optimization 
To develop a low-cost and ready-to-use ATP biosensor we selected a paper-based format since paper is economical, environment friendly, allows passive transport of liquids and is compatible with many chemicals. We decided to exploit the exquisite sensitivity of firefly luciferase towards ATP and developed a new straightforward lyophilization procedure to obtain a freeze-dried "nanolantern" comprising the luciferin-luciferase system on nitrocellulose paper. Conventional lyophilization protocols include a preliminary freezing step, a primary drying and a secondary drying processing. The first step is generally carried on for 10-30 min, depending on total volume, at temperatures between -30 and $-80^{\circ} \mathrm{C}$ to remove water molecules. We optimized a protocol in which no preliminary freezing step is included and freezing occurs directly during the freeze-drying process. While during conventional lyophilization we clearly observed that the lyophilized luciferase/D-luciferin was detached from the paper, using the alternative protocol, the lyophilized luciferase/D-luciferin reagents result perfectly attached to the paper disc (Fig. S1a). Moreover, the effect of addition of different amounts of cryoprotectant R18 medium $(0,1,2,3$, and $4 \mu \mathrm{l})$ to the lyophilization mix was evaluated. Addition of $2 \mu \mathrm{L}$-volume of R18 to the lyophilization mix, provided the highest BL signal after reconstitution with $8 \mu \mathrm{l}$ of $\mathrm{DB}$ and addition of a $2 \mu l$-volume of $1.65 \mathrm{mM}$ ATP solution in DB, demonstrating optimal protection of the enzyme catalytic activity during the lyophilization procedure (Fig.S1b) Also reconstitution buffer selection was investigated and the reconstitution buffer (DB) provided in the ATP Bioluminescence Assay Kit HS II from Roche provided the best results, showing $36 \pm 6 \%$ increase in the BL signal 10min after ATP addition (1.65 mM ATP solution in DB) in comparison to reconstitution with bidistilled $\mathrm{H}_{2} \mathrm{O}$ (Fig.S1c).

We then evaluated the impact of lyophilization and reconstitution processes on luciferase emission properties and stability. The ATP sensing paper was characterized with benchtop luminometer by inserting the $5 \mathrm{~mm}$ nitrocellulose discs into the wells of a black 96-well microtiter plate. BL intensities were recorded after the reconstitution of lyophilized discs with $8 \mu$ l-volume of DB and the addition of $2 \mu \mathrm{L}$ of $1.65 \mathrm{mM}$ ATP. As show in Fig 2a a bioluminescence maximum of $555 \mathrm{~nm}$ was obtained with a half bandwidth of $75 \mathrm{~nm}$. This behaviour is consistent with wild type $P$. pyralis luciferase 
emission previously reported (Branchini et al., 2004). By analysing BL signals obtained with benchtop instrumentation and smartphone Samsung Galaxy S7, where BL intensities were measured every 10 min using an integration time of $10 \mathrm{sec}$ with ISO 800, emission kinetics showed a glow-type behaviour with a maximum signal at $14 \mathrm{~min}$ after ATP addition(Fig.2b). This is due to the presence of sucrose in the R18 cryoprotectant solution which, in addition to protect the enzymatic activity during lyophilization, promotes higher structural stability of the firefly luciferase with consequent controlled release of light energy (Rasouli et al., 2011). This is in contrast to flash-type emission kinetics of the ATP Bioluminescence Assay Kit HS II (Roche) related to the high concentration of luciferase in the assay. The glow-type emission behaviour greatly simplifies the integration of this "nanolantern" into a portable light detector. An optimal acquisition time window was identified between 6 and $22 \mathrm{~min}$ in which the mean BL signal is $90 \pm 10 \%$ of the maximum emission.

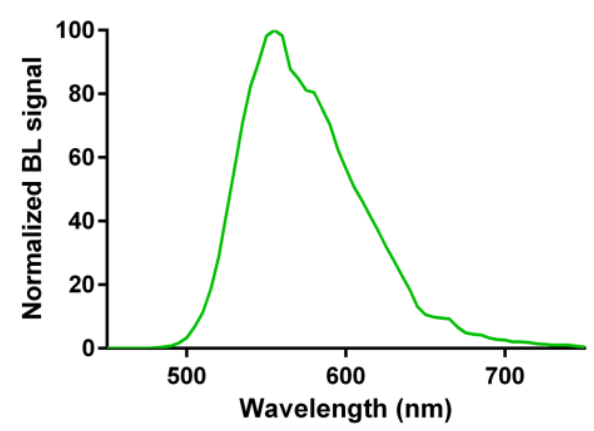

b

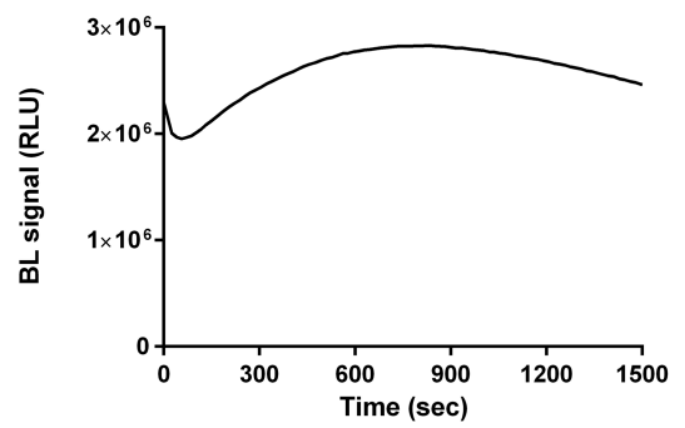

Fig. 2. Characterization of ATP sensing paper: (a) Normalized BL emission spectrum $(\lambda \max =555$ $\mathrm{nm}$ ) and (b) emission kinetic of ATP sensing paper obtained with Varioskan Flash multimode reader after the reconstitution of LR with $8 \mu \mathrm{L}$ of DB and the addition of $2 \mu \mathrm{L}$ ATP solution $1.65 \mathrm{mM}$.

Analytical performance of the ATP sensing paper

After the preliminary characterization of the ATP sensing paper, we optimized the assay conditions and evaluated its analytical performance by detecting ATP in a dose-dependent manner. To this end, 
$2 \mu \mathrm{L}$-volumes of ATP solutions in the range of $1.64 \times 10^{-3}-1.64 \times 10^{-9} \mathrm{M}$, corresponding to $3.3 \times 10^{-}$ ${ }^{9}-3.3 \times 10^{-15}$ moles of ATP, were analyzed with the ATP sensing paper.

In optimized conditions the assay procedure is very straightforward, consisting in just a few steps: a volume of $8 \mu \mathrm{L}$ of either sample or $2 \mu \mathrm{L}$ ATP solution are incubated with the ATP sensing paper for $10 \mathrm{~min}$ at room temperature $\left(22^{\circ} \mathrm{C}\right)$. Then acquisition is performed with Oneplus 5 camera integrating BL signals for $30 \mathrm{sec}$ with ISO 3200. To optimize the acquisition of BL signals with OnePlus 5 camera, we imaged ATP sensing paper using $30 \mathrm{sec}$ of exposure time and ISO values from 1600 to 3200 (Fig. 3). Although aberrant images were obtained at higher concentration of ATP using ISO 3200, higher ISO setting are required for detecting lower concentrations of ATP. According to these results, we selected an integration time of $30 \mathrm{sec}$ with ISO 3200 setting to achieve the best sensitivity for our assay.

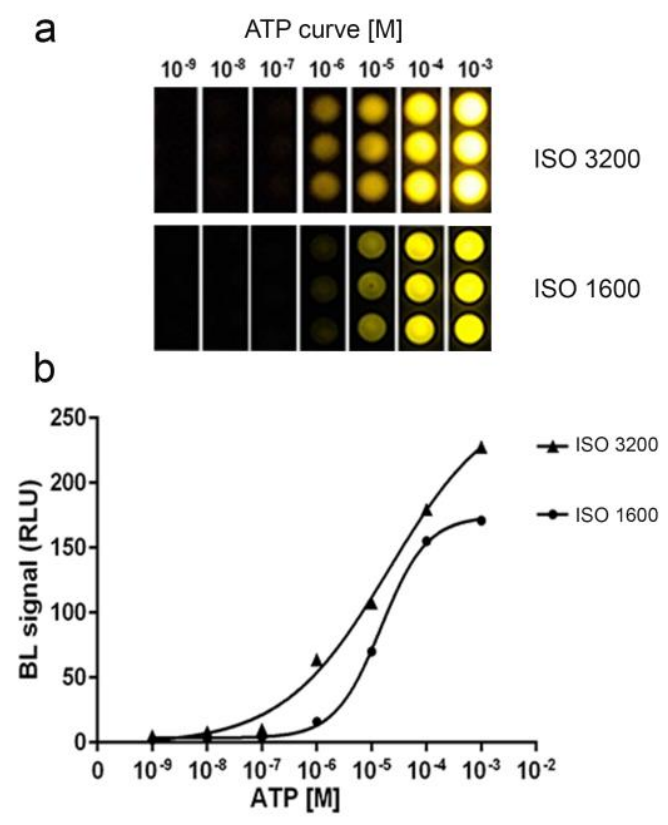

Fig. 3. ATP calibration curves achieved by ATP sensing papers: (a) BL images obtained with OnePlus 5 smartphone camera at 1600 and 3200 ISO settings (30-s acquisition time) after the addition of $2 \mu \mathrm{L}$ of the indicated ATP concentrations and (b) graphical elaboration of BL signals with Image J Software. 
In addition, for in-house comparison of the analytical performance of the ATP sensing paper we tested two smartphone cameras, Oneplus 5 and Samsung Galaxy S7 integrated cameras, selected for their built-in ProCam application enabling manual selection of parameters including shutter-speed up to $30 \mathrm{sec}$ and ISO settings values up to 3200 for low-light applications. To demonstrate the ATP sensing paper capabilities and to assess the feasibility of the different smartphones integrated CMOS for BL light detection, we employed maximum performance settings of $30 \mathrm{sec}$ with ISO 3200 for OnePlus 5 and $10 \mathrm{sec}$ with ISO 800 for Samsung Galaxy S7. Dose-response curves for ATP are shown in Fig. 4. Under the optimized conditions, the BL signal is proportional to the ATP concentration showing a limit of detection (LOD) of $2.1 \times 10^{-12}$ moles of ATP with Samsung Galaxy S7 and a LOD of $3.8 \times$ $10^{-14}$ moles of ATP with OnePlus 5 camera. This is in agreement with Kim et al., 2017 who performed an inter-phone performance comparison to evaluate the integrated cameras of Galaxy S4, Galaxy Note 3, LG G2, Oneplus, and iPhone $5 \mathrm{~S}$ in a standardized test and in a maximum-performance test. In fact, despite the performance order was $\mathrm{G} 2>$ iPhone $5 \mathrm{~S}>$ Note $3>\mathrm{S} 4>$ Oneplus in the standardized test, when the test was performed in the best performance setup for each phone, the Oneplus was superior thanks to the lower signal background from the CMOS sensor. The feasibility of using the ATP sensing paper with a cooled CCD portable camera, was also investigated. A LOD of $1.7 \times 10^{-13}$ moles of ATP was obtained using the ATIK 383L. Concerning detectability, while the benchtop luminometer Varioskan Flash was able to quantify a concentration of ATP two decades lower $\left(10^{-15}\right.$ moles of ATP). LODs obtained with the ATIK 383L CCD and OnePlus 5 cameras were respectively one and two orders of magnitude lower than those obtained with Samsung Galaxy S7. Thanks to the significant improvement of the LOD, these results confirmed the suitability of OnePlus 5 camera for quantitative detection of ATP present in biological fluids at fmoles levels, which is consistent with values of clinical interest. 


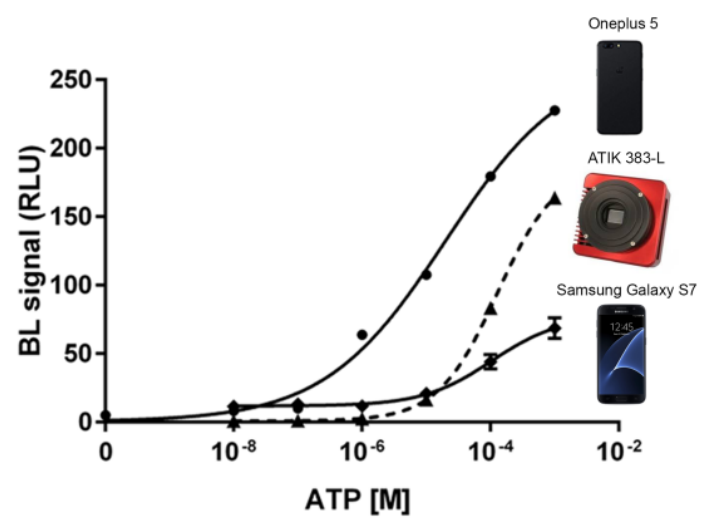

Fig. 4: Comparison of ATP calibration curves obtained with ATP sensing papers using different portable light detectors: Samsung Galaxy S7 (solid line), OnePlus 5 camera (solid line) and cooled CCD ATIK 383L- camera (dotted line). ATP sensing paper were reconstituted with $8 \mu \mathrm{L}$ of DB and incubated for about 10 min with $2 \mu \mathrm{L}$ of the indicated ATP concentrations. BL measurements were obtained using the maximum performance settings of the three portable light detectors: $30 \mathrm{~s}$ at ISO 3200 for OnePlus 5 smartphone, $10 \mathrm{~s}$ at ISO 800 for Samsung Galaxy S7 smartphone and $60 \mathrm{~s}+5^{\circ} \mathrm{C}$ for CCD ATIK 383L camera. BL images were elaborated by ImageJ Software. Error bars are present but not visible due to the minimum variation of BL signals obtained between triplicates.

\section{Stability and reproducibility of the ATP sensing paper}

In an effort of obtaining a stable ready-to-use ATP-paper based biosensor, we assessed emission properties and stability of several ATP sensing papers stored at different temperature conditions and for different periods of time.

We first evaluated the stability of the ATP sensing paper stored at room temperature $\left(25^{\circ} \mathrm{C}\right),+4^{\circ} \mathrm{C}$ and $-20{ }^{\circ} \mathrm{C}$ for $48 \mathrm{~h}$. Using the standard procedure to obtain ATP sensing paper, after $48 \mathrm{~h}$ the enzymatic activity showed a decrease in BL signals of about $31 \pm 5 \%$ and $26 \pm 5 \%$ at $+4{ }^{\circ} \mathrm{C}$ and $20^{\circ} \mathrm{C}$, respectively (Fig. S2). An analogous BL decrease was observed during the storage at $+4^{\circ} \mathrm{C}$ of the ATP sensing paper obtained with the new procedure (BL signal decrease of $35 \pm 5 \%$ ). At room temperature the ATP sensing paper obtained with both lyophilization procedures showed a significant loss of enzymatic activity (about $95 \pm 2 \%$ ). Conversely, after storage at $-20^{\circ} \mathrm{C}$, evaluated in a 80 
days-period of time, we observed a drop of the BL signal of about $10 \pm 8 \%$ and $15 \pm 5 \%$ after 20 and 80 days, respectively (Fig. 5b).

To test the reproducibility of the ATP sensing paper and the feasibility of implementing this biosensor in different laboratories and settings, a preliminary intra- and inter laboratory validation of the ATP sensing paper was assessed by testing a total of 20 different ATP sensing papers obtained in two different laboratories.

ATP sensing papers were obtained in different laboratories using different lyophilizers following the same reagents, procedure and protocols optimized in the Institut Catala de Nanociencia i Nanotecnologia ICN2, Bellaterra, Barcellona, Spain. ATP solutions were analyzed in the concentrations range of $1.64 \times 10^{-3}-1.64 \times 10^{-9} \mathrm{M}$ and measured with the same smartphone (Samsung Galaxy S7) with same settings (integration for $10 \mathrm{sec}$ with ISO 800) (Fig. 5a). The results show a comparable LODs with coefficient of variation $(\mathrm{CVs})$ of $16 \pm 3 \%$ confirming the robustness of the alternative freeze-drying procedure.

To demonstrate the suitability of ATP sensing paper for practical applications, an intra-laboratory validation was performed in the Department of Chemistry "Giacomo Ciamician", Bologna, Italy to evaluate the content of ATP in bacterial cells. Indeed, analyzing the standard curve obtained with serial ATP dilutions in DB, we reported a strong correlation between cell number and moles of ATP detected with Oneplus 5 smartphone. An optimized protocol to concentrate cells was required to obtain a lysed bacterial solution of $10^{3}-10^{7} \mathrm{CFU}$ in $10 \mu \mathrm{L}$ corresponding to $10^{-15}-10^{-11}$ moles of ATP. In optimized conditions we were able to detect 4 x $10^{4}$ bacterial cells with Oneplus 5 smartphone (30 sec, ISO 3200). This is consistent with the threshold for positivity of UTI reported in the literature $\left(10^{5} \mathrm{CFU} / \mathrm{mL}\right)$.

When comparing the analytical performance of the ATP sensing paper combined with Oneplus 5 smartphone detection, the LOD $\left(10^{-14}\right.$ moles of ATP corresponding to $\left.3.8 \times 10^{4} \mathrm{CFU} / \mathrm{mL}\right)$ was about one order of magnitude higher compared to those recently reported by Nguyen et al. (2018) and Santangelo et al. (2018). However, it must be pointed out that our assay contains less amount of 
reagents than previously reported ATP biosensors relying on D-luciferin/luciferase system and does not require the use of benchtop luminometer for BL acquisition but exploits the smartphone camera as portable optical detector (Nguyen et al. 2018). Our ATP sensing paper format provides a total of about 4160 assays using reagents from a single kit, compared to only 416 assays that are obtained by other authors (Nguyen et al., 2018) and 1000 assays in microwell plate format according to the manufacturer's protocol. Thus, our approach is more cost-effective, with an average cost per sample ( 0.5 eurocents) between 5 and 11 times lower compared to previously reported assays and biosensors. Furthermore, the use of a smartphone camera as a portable light detector further reduces the costs of analysis associated with the use of expensive equipment such benchtop luminometer facilitating the analysis procedure. The replacement of wild-type luciferases with new chimeric luciferases with lower $\mathrm{K}_{\mathrm{m}}$ value for ATP and high specific activity will be addressed to further improve the analytical performance of the ATP sensing paper (Branchini et al., 2017).

a

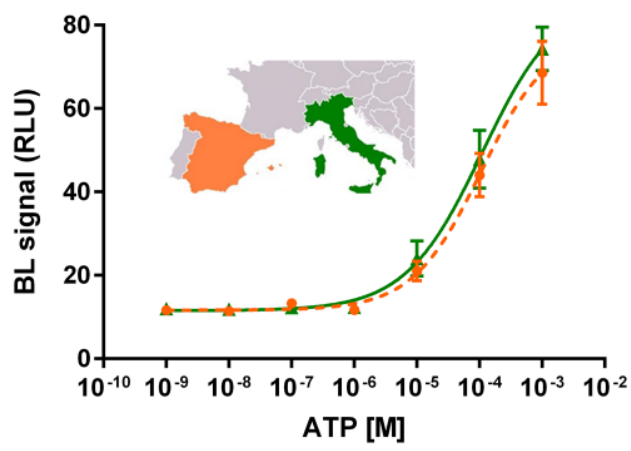

b

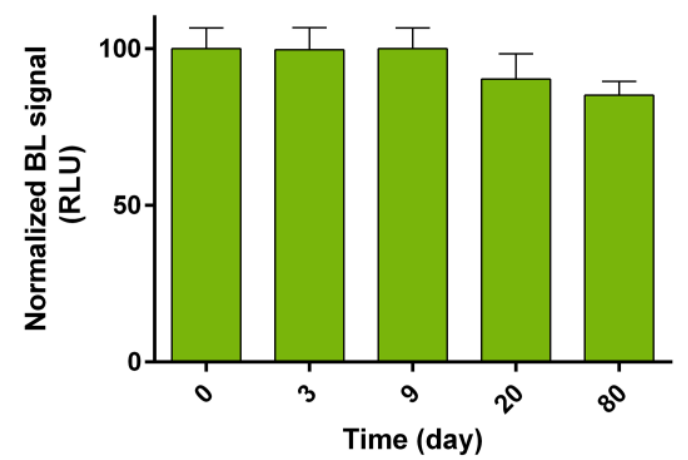

Fig. 5: Fig.

5: (a) Inter-laboratory validation: ATP calibration curve obtained with ATP sensing papers lyophilized with two freezy-dry instrumentations, one located at Department of Chemistry "Giacomo Ciamician", University of Bologna, Italy (solid line) and one located at Institut Català de Nanociència i Nanotecnologia (ICN2), Bellaterra, Spain (dotted line). Both experiments were produced by the same analyst and BL signals were acquired with smartphone camera (Samsung Galaxy S7) for $10 \mathrm{~s}$ at ISO 800. (b) Stability of ATP sensing paper achieved with alternative procedure and stored at $20^{\circ} \mathrm{C}$. Each indicated day (from 0 to 80 days), triplicate ATP sensing papers (obtained in the same 
lyophilization session) are reconstituted with $8 \mu \mathrm{L}$ of DB and BL measurements were obtained after the addition of $2 \mu \mathrm{L}$ of ATP $1.65 \mathrm{mM}$. BL signals are acquired with Samsung Galaxy S7 (10 s at ISO 800) and analyzed with ImageJ software. BL signals are normalized with respect to day 0 (ATP sensing paper immediately reconstituted after the end of the alternative lyophilization procedure).

\section{Real samples analysis}

The sensitivity of the ATP sensing paper corroborates its use for diverse applications in which the detection of living cells can be assessed by ATP, such as the detection of antibiotic effects on bacteria. To test the proposed smartphone-based biosensor integrating lyophilized LR on real samples we evaluated the ATP concentration in urine spiked samples as a screening test for urinary infections. As proof-of concept, exploiting measurement ATP as metabolic activity of microbial contamination, we used ATP sensing paper for determining urinary tract infection (UTI) in which the threshold for positivity is $10^{5} \mathrm{CFU} / \mathrm{mL}$ (Schmiemann et al., 2008). For proving the feasibility to detect UTI in biological samples, fresh urine samples from an healthy volunteer were spiked with $E$. coli cells to achieve a concentration of $10^{5} \mathrm{CFU} / \mathrm{mL}$, corresponding to asymptomatic bacteriuria in an individual without urinary tract symptoms (https://uroweb.org/wp-content/uploads/19-Urologicalinfections 2017 web.pdf). Since urine $\mathrm{pH}$ is strongly influenced by metabolic disorders, infections and other physio-pathological conditions, a very simple pretreatment of the sample was necessary to avoid aspecific effects on the luciferase/ D-luciferin reaction. A preconcentration step was thus introduced, and before bacteria lysis to replace the complex urine matrix with $\mathrm{DB}$. For this reason, $10 \mathrm{~mL}$ of sample were harvested by centrifugation and the pellet resuspended with $50 \mu \mathrm{L}$ of DB. Thanks to its selectivity to disrupt membrane of bacterial cells, B-PER Bacterial Extraction Reagent $(50 \mu \mathrm{L})$, was used to extract ATP from E. coli cells. Different incubation times (5, 10, 15, 20 and 30 min), different temperatures $\left(22^{\circ} \mathrm{C}\right.$ and $\left.37^{\circ} \mathrm{C}\right)$ under or without orbital shaking were tested in order to select the experimental conditions (Fig. S3) providing the most efficient lysis. In optimized conditions an incubation time of $10 \mathrm{~min}$ at $22^{\circ} \mathrm{C}$ without orbital shaking was select to extract ATP from E. coli cells. The cellular ATP levels were then quantified after the addition of the cell lysate $(10 \mu \mathrm{L})$ on 
ATP sensing paper. BL image obtained with the Oneplus 5 camera provided a direct visual estimation of E. coli concentration (Fig. 6). Fig. 6a shows a detailed BL image corresponding to the ATP calibration curve and to the ATP released by the spiked sample (circled in red). BL signals were analyzed with ImageJ Software and the value obtained by the real sample was interpolated to the ATP calibration curve (red triangle) (Fig. 6b). By interpolating the signal of the sample in the ATP calibration curve we obtained an ATP content of $10^{-13}$ moles of ATP, corresponding to $10^{5} \mathrm{CFU} / \mathrm{mL}$ of E. coli.

a

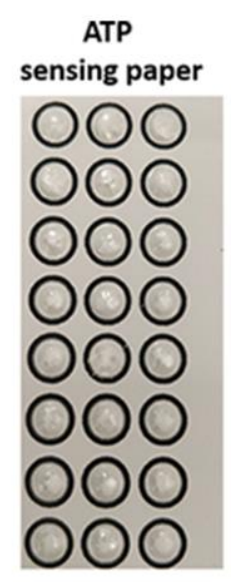

ATP calibration curve (Moles)

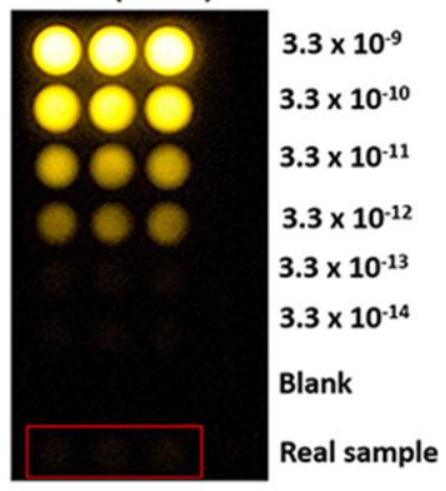

b

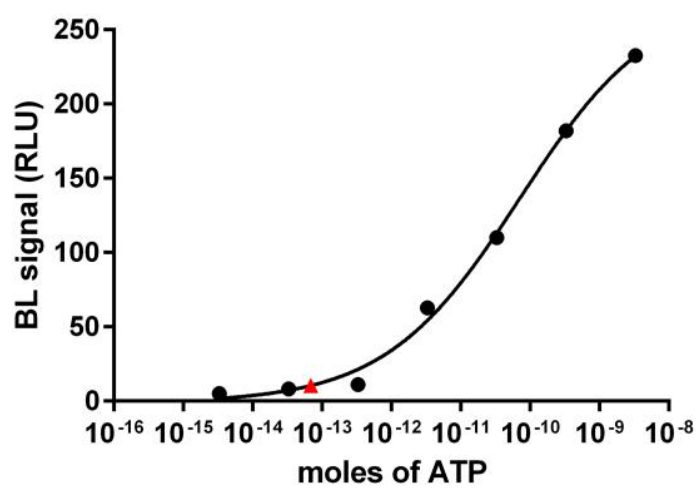

Fig. 6: (a) Picture of the ATP sensing paper obtained lyophilizing LR with alternative freeze-drying procedure. Each ATP sensing paper can be used to analyze a real sample in triplicate. It contains 7 x 3 microwells used for ATP calibration curves (concentration range from $1.64 \times 10^{-3}$ to $1.64 \times 10^{-15} \mathrm{M}$ ) and 3 microwells for sample testing and corresponding BL image obtained with OnePlus 5 smartphone; (c) Results obtained after image elaboration with Image J Software. The BL signal obtained from ATP content of $10^{5} \mathrm{CFU} / \mathrm{mL}$ E. Coli. real sample was interpolated to the ATP calibration curve. In the designed configuration $8 \times 3$ circular areas are used to evaluate the ATP content in $10^{5} \mathrm{CFU} / \mathrm{mL}$ of E. coli. 


\section{Conclusion}

For the first time, we demonstrated the possibility to obtain a low-cost bioluminescent ATP sensing paper based on luciferase/D-luciferin reaction that exploits smartphone camera as detector. The proposed biosensor consists of LR lyophilized on nitrocellulose paper obtained with a new alternative freeze-drying procedure and smartphone for bioluminescence detection. As proof-of-concept, we developed an assay to detect ATP levels as indicator of metabolic activity of E. coli cells in liquid samples. The suitability of the paper-based sensing platform for detecting ATP concentration in urine samples was investigated. Indeed, the reported data confirm the suitability of using this approach to address the detection of ATP. In addition, ad hoc application can be developed and implemented for data elaboration. The use of a newly developed chimeric luciferase with lower $\mathrm{K}_{\mathrm{m}}$ value for ATP and high specific activity could improve the analytical performance of the ATP sensing paper, opening new opportunities for detecting bacterial pollution level (Tanii et al. 2001) and for analysing microbial content in drinking water, food safety and public hygiene (Champiat et al., 2001) that generally need specialized laboratories with highly qualified personnel and expensive equipment.

\section{Acknowledgements}

This research was sponsored in part by PRIN 2015 "Multifunctional nanotools for advanced cancer diagnostics" (Prot. 2015TWP83Z) and the NATO Science for Peace and Security Programme under Grant No. 985042. The ICN2 is funded by the CERCA Programme / Generalitat de Catalunya and also supported by the Severo Ochoa program of the Spanish Ministry of Economy, Industry and Competitiveness (MINECO, grants No. SEV-2017-0706 and MAT2017-87202-P). 


\section{References}

1. Adachi, Y., Sugawara, M., Taniguchi, K., Umezawa, Y., 1993. Anal. Chim. Acta, 281, 577.

2. Akbara, M., Essab, M.M., Daradkehb, G., Abdelmegeeda, M.A., Choia, Y., Mahmoodd, L., Songa,B-J, 2016. Brain Res. 1637, 34-55.

3. Álvarez-Diduk, R., Orozco, J., Merkoçi, A. 2017. Sci. Rep. 7, 976.

4. Arduini, F., Cinti, S., Caratelli, V., Amendola, L., Palleschi, G., Moscone, D., 2019. Biosens Bioelectron. 126, 346-354.

5. Aycicek, H., Oguz, U., Karci, K., 2006. Int. J. Hyg. Environ.-Health. 209, 197-201.

6. Branchini, B.R., Ablamsky, D.M., Murtiashaw, M.H., Uzasci, L., Fraga, H., Southworth, T.L., 2007. Anal Biochem. 361(2), 253-262.

7. Branchini, B.R., Behney, C.E., Southworth, T.L., Fontaine, D.M., Gulick, A.M., Vinyard, D.J., Brudvig, G.W., 2015. J. Am. Chem. Soc. 137, 7592-7595.

8. Branchini, B.R., Southworth, T.L., 2017. Methods Enzymol. 589, 351-364.

9. Branchini, B.R., Southworth, T.L., Fontaine, D.M., Kohrt, D,, Welcome, F.S., Florentine, C.M., Henricks, E.R., DeBartolo, D.B., Michelini, E., Cevenini, L., Roda, A, Grossel, M.J., 2017. Anal Biochem. 534,36-39.

10. Branchini, B.R., Southworth, T.L., Murtiashaw, M.H., Magyar, R.A., Gonzalez, S.A., Ruggiero, M.C., Strohm J.G., 2004. Biochemistry. 43,7255-7262.

11. Calabria, D., Caliceti, C., Zangheri, M., Mirasoli, M., Simoni, P., Roda, A. 2017. Biosens Bioelectron 94, 124-130

12. Capoferri, D., Álvarez-Diduk, R., Del Carlo, M., Compagnone, D., Merkoçi, A., 2018. Anal. Chem. 90, 5850-5856

13. Carrea, G., Bovara, R., Mazzola, G., Girotti, S., Roda, A., Ghini, S., 1986. Anal. Chem. $58,331-333$. 
14. Cevenini, L., Calabretta, M.M., Lopreside, A., Tarantino, G., Tassoni, A., Ferri, M., Roda, A., Michelini, E., 2016. Anal Bioanal Chem. 408, 8859-68

15. Champiat, D., Matas, N., Monfort, B., Fraass, H., 2001. Luminescence. 16, 193-198.

16. Dostálek, P., Brányik, T., 2005.Czech J. Food Sci. 23, 85-92.

17. EAU Guidelines on Urological Infections. European Association of Urology. http://www.uroweb.org/nc/professional-resources/guidelines/online/

18. Esimbekova, E.N., Torgashina, I.G., Kratasyuk, V.A., 2007. Biochemistry (Moscow), 74, 695-700.

19. Feng, S., Dong, T., Yang, Z., 2014.Conf Proc IEEE Eng Med Biol Soc.; 3114-3117.

20. Ge, L., Wang, P., Ge, S., Li, N., Yu, J., Yan, M., Huang, J., 2013. Anal. Chem. 85, 39613970.

21. Huang, Y.S., Chen, Y.C., Chen, M.L., Cheng, A., Hung, I.C., Wang, J.T., Sheng, W.H., Chang, S.C., 2015. Am J Infect Control., 43, 882-886.

22. Ivančić, V., Mastali, M., Percy, N., Gornbein, J., Babbitt, J.T., Li, Y., Landaw, E.M., Bruckner, D.A., Churchill,B.M., Haake, D.A, 2008. J Clin Microbiol. 46, 1213-1219.

23. Kim, H., Jung, Y., Doh, I.J., Lozano-Mahecha, R.A., Applegate, B., Bae, E., 2017. Sci Rep.7, 40203.

24. Kratasyuk, V. A. and Esimbekova, E. N., 2003. Polymer immobilized bioluminescent systems for biosensors and bioinvestigations. In: R. Arshady (Ed.), Polymeric biomaterials, The PBM Series (Introduction to Polymeric Biomaterials), London, Vol. 1, pp. 301-343.

25. Lonshakova-Mukina, V., Esimbekova, E., Kratasyuk, V., 2015. Sens. Actuators B: Chem. $213,244-247$.

26. López-Marzo, A.M., Merkoçi, A, 2016. Lab Chip. 16, 3150-3176. 
27. Lopreside, A., Calabretta, M.M., Montali, L., Ferri, M., Tassoni, A., Branchini, B.R., Southworth, T., D’Elia, M., Roda, A., Michelini, E., 2019. Anal Bioanal Chem. (DOI: $10.1007 / \mathrm{s} 00216-019-01805-2)$

28. Lundin, A. VTT Symp. 1999, 193, 337

29. Lundin, A., Hallander, H., Kallner, A., Lundin, U.K., Osterberg, E., 1989. J Biolumin Chemilumin. 4, 381-389.

30. Michelini, E., Calabretta, M.M., Cevenini, L., Lopreside, A., Southworth, T., Fontaine, D.M., Simoni, P., Branchini, B.R., Roda, A., 2019. Biosens Bioelectron. 123, 269-277.

31. Morciano, G., Sarti, A.C., Marchi, S., Missiroli, S., Falzoni, S., Raffaghello, L., Pistoia, V., Giorgi, C., Di Virgilio, F., Pinton, P., 2017. Nat Protoc. 12, 1542-1562.

32. Nascetti, A., Mirasoli, M., Marchegiani, E., Zangheri, M., Costantini, F., Porchetta, A., Iannascoli, L., Lovecchio, N., Caputo, D., de Cesare, G., Pirrotta. S., Roda, A., 2019. Biosens Bioelectron.123, 195-203.

33. Nguyen, D. T., Kim, H.Ri., Jung, J.H., Lee, K-B, Kim, B.C., 2018. Sens. Actuators B: Chem. 260, 274-281.

34. Onyango, I.G., Dennis, J., Khan, S.M., 2016. Aging Dis. 7, 201-214.

35. Raouli, S., Hosseinkhani, S., Yaghmaei, P., Ebrahim-Habib, A, 2011. Appl Biochem Biotechnol. 165, 572-582.

36. Ribeiro, A. R., Santos, R. M., Rosario, L. M., Gil, M. H., 1998. J. Biolumin. Chemilumin. 13,371 .

37. Roda, A., Pasini, P., Mirasoli, M., Michelini, E., Guardagli, M., 2004. Trends.Biotechnol. $22,295-303$.

38. Santangelo, M.F., Libertino, S., Turner, A.P.F., Filippini, D., Mak, W.C., 2018. Biosens Bioelectron. 99,464-470.

39. Satoh, T., Kato, J., Takiguchi, N., Ohtake, H., Kuroda, A., 2004. Biosci. Biotechnol. Biochem. 68, 1216-1220. 
40. Schmiemann, G., Kniehl, E., Gebhardt, K., Matejczyk,M.M, Eva Hummers-Pradier, E., 2010. Dtsch Arztebl Int. 107, 361-367.

41. Squirrell, D.J., Price, R.L., Murphy, M.J., 2002. Anal. Chim. Acta. 457, 109-114.

42. Tanii, T., Goto, T., Iida, T., Koh-Masahara, M., Ohdomari, I., 2001. Jpn. J. Appl. Phys., 40.

43. Thore, A., Lundin, A., Anséhn, S., 1983. J Clin Microbiol. 17, 218-224.

44. Ugarova, N.N., Brocko, L.Yu., Kost, N.V., 1982. Enzyme Microb. Technol. 4, 224-228.

45. Wang, W., Zhao, Q., Luo, M., Li, M., Wang, D., Wang, Y., Liu, Q, 2015. ACS Appl Mater Interfaces. 7, 20046-20052.

46. Wu, M., Lai, Q., Ju, Q., Li, L., Yu, H.D., Huang, W., 2018. Biosens Bioelectron. 102, 256-266.

47. Yamana, K., Ohtani, Y., Nakano, H., Saito, I., 2003. Bioorg. Med. Chem. Lett., 13, 3429.

48. Yousefi-Nejad, M., Hosseinkhani, S., Khajeh, K., Ranjbar B., 2007. Enzyme Microb. Tech. 40, 740-746.

49. Zhang, C., Rissman, R.A., Feng, J., 2015. J Alzheimers Dis. 44, 375-378.

50. Zhang, Z., Wang, J., Ng, R., Li, Y., Wu, Z., Leung, V., Imbrogno, S., Pelton, R., Brennan, J.D., Filipe, C.D., 2014. Analyst. 139, 4775-4778. 